\title{
Cerebral Infarction Associated With Thrombosed Developmental Venous Anomaly: A Case Report
}

Haniyeh Javanmardi ${ }^{1}$, M ehdi karimian ${ }^{2}$, Afshin Borhani Haghighi ${ }^{3 *}$

${ }^{1}$ Clinical neurology Research Center, Student Research Committee, Shiraz University of M edical Sciences, Shiraz, Iran.

${ }^{2}$ Clinical Neurology Research Center, Shiraz University of M edical Sciences, Shiraz, Iran.

${ }^{3}$ Clinical Neurology Research Center, Department of Neurology, Shiraz University of Medical Sciences, Shiraz, Iran.

*Corresponding Author: Email: Aborhani@sums.ac.ir

\section{Introduction:}

Developmental venous anomaly (DVA, venous angioma) is a congenital vascular variant of cerebral venous drainage, which consists of several radial veins draining into an enlarged central vein (caput medusa appearance). This anatomical variations are usually followed by benign and asymptomatic clinical course, so it is incidentally detected at M RI, M RA, CT, angiography or autopsy performed for unrelated problems. Rarely DVAs become symptomatic and present with headache, seizures, numbness, diplopia, paresthesia, syncope and focal neurologic deficit secondary to thrombosis in drainage veins. Hemorrhagic complications occur more often than isolated ischemic events. Also, non-hemorrhagic brain infarction is a rare complication in these patients. We describe a patient with non-hemorrhagic venous infarction associated with DVA.

\section{Case report:}

A 57-year- old female patient was admitted to Namazi hospital with complaints of acute severe headache in the right temporo-occipital region, vertigo, left sided paresthesia and weakness. She was in good health a week prior to admission when the blurred vision appeared in her right eye. The patient had previous history of recurrent episodes of migraine-like headache that controlled by medical therapy. The family history was unremarkable. Her only medications were various oral contraceptives and propranolol. On examination, she was afebrile and alert with stable vital signs. Her pupils were equal and reactive to light. Neurological examination was normal and the cranial nerves were intact. The deep tendon reflexes were brisk and symmetric and bilateral Babinski and Hoffmann's signs were present. On admission, her speech, memory, and intellectual performance were normal. She had weakness of the upper and lower extremities (especially on the left side) and her knees were unstable while walking. Hematology and coagulation tests (protein $\mathrm{C}$, activated protein $\mathrm{C}$ resistance, protein $\mathrm{S}$, homocysteine, anticardiolipin antibodies, antithrombin III and antinuclear anti body) were normal.

The initial CT scan of the brain demonstrated no evidence of abnormal density, hydrocephalus or hemorrhagic process in the cerebral hemispheres, M RI findings revealed several radially arranged veins converging to a small enlarged vein in the right temporo-occipital lobe. Thrombosis of collector veins detected as a hypersignality on contrast enhanced M RI. Also, "caput medusa" configuration was detectable in angiography.

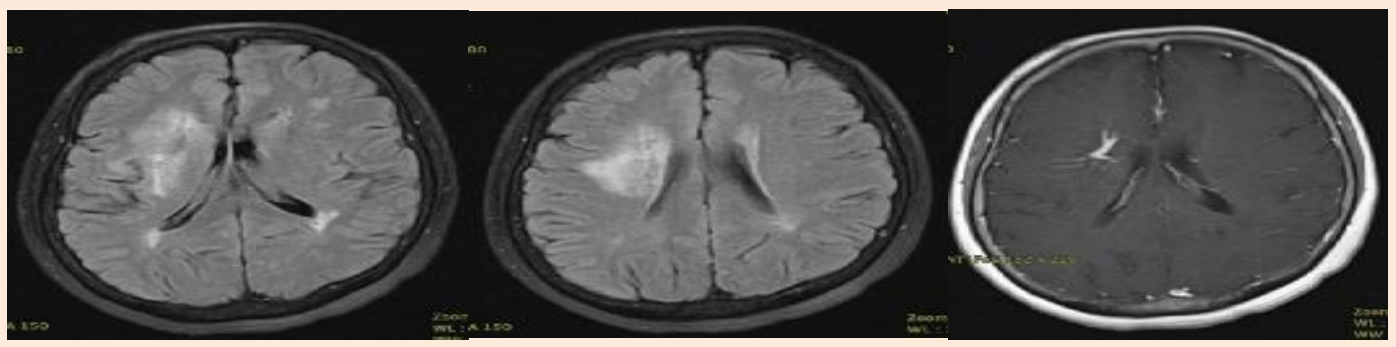

DOI: $10.7575 /$ aiac.abcmed.ca1.14

A I Published Date: February 2017

Peer-review is under responsibility of the 9th Iranian Stroke Congress.

Published by Australian International Academic Centre, Australia

This published work is open access under the CC BY license.

Available online at www.abcmed.aiac.org.au 